\title{
Age Prediction using Image Dataset using Machine Learning
}

\author{
Ishita Verma, Urvi Marhatta, Sachin Sharma, Vijay Kumar
}

\begin{abstract}
Gender is a central feature of our personality still. In our social life it is also an significant element. Artificial intelligence age predictions can be used in many fields, such as smart human-machine interface growth, health, cosmetics, electronic commerce etc. The prediction of people's sex and age from their facial images is an ongoing and active problem of research. The researchers suggested a number of methods to resolve this problem, but the criteria and actual performance are still inadequate. A statistical pattern recognition approach for solving this problem is proposed in this project.Convolutionary Neural Network (ConvNet / CNN), a Deep Learning algorithm, is used as an extractor of features in the proposed solution. CNN takes input images and assigns value to different aspects / objects (learnable weights and biases) of the image and can differentiate between them. ConvNet requires much less preprocessing than other classification algorithms. While the filters are hand-made in primitive methods, ConvNets can learn these filters / features with adequate training.In this research, face images of individuals have been trained with convolutionary neural networks, and age and sex with a high rate of success have been predicted. More than 20,000 images are containing age, gender and ethnicity annotations. The images cover a wide range of poses, facial expression, lighting, occlusion, and resolution.
\end{abstract}

Keywords: Facial Images; Gender Prediction; Age Prediction; Convolutional Neural Network; Deep Learning.

\section{INTRODUCTION}

The aim is to predict the age of individuals using image data sets. An growing number of applications, especially after the increase in social networks and social media, are being concerned with automatic age classification. Age and gender are the two most fundamental facial qualities in social interaction. In smart applications, such as access control, human computer interaction, enforcement, marketing intelligence and visual supervision, etc, it is important to make age evaluations using one facial image.Machine learning: supervised learning, image recognition, and deep learning: a groundbreaking neural network and profound learning are the most common technologies used in this project. Supervised learning can be described as a machine learning technique in which the input is mapped to the output using input-output pair training data. TensorFlow is an open-source library used for math, data flow and specific machine learning applications.

Revised Manuscript Received on October 20, 2019.

Ishita Verma, Department of Computer Science and Engineering, Graphic Era Deemed to be University, Dehradun, India.

Urvi Marhatta, Department of Computer Science and Engineering, Graphic Era Deemed to be University, Dehradun, India.

Sachin Sharma, Department of Computer Science and Engineering, Graphic Era Deemed to be University, Dehradun, India.

Vijay Kumar, Department of Physics, Graphic Era Hill University, Dehradun India.
Convolutional Neural Network $(\mathrm{CNN})$ is one of the most prevalent algorithms that has gained a high reputation in image feature extraction.Age Classification using Convolutional Neural Networks:

A Convolutionary Neural Network (ConvNet / CNN) is a Deep Learning algorithm, which allows an input image to take on different aspects / objects and can be distinguished from one image (learnable weights and biases). ConvNet requires much less preprocessing than other classification algorithms. While the filters are hand-made in primitive methods, ConvNets can learn these filters / features with adequate training. The ConvNet architecture is similar to that of neurons in the human brain and was influenced by the Visual Cortex organisation. Within a limited area of the visual field known as the Receptive Field, only individual neurons respond to stimuli. The entire visual area is protected by a selection of these fields. About Dataset: a broad facial dataset of long age (range 0-116 years old) is a UTKFace data set. The data collection consists of more than 20,000 facial images with age, gender and ethnicity annotations. The images cover a wide range of poses, facial expression, lighting, occlusion, resolution .It can be used for a variety of tasks, for example face detection, age estimation, age progression, position of landmarks etc.

The survey is focused on the age detection of the neural network $(\mathrm{CNN})$ image dataset architecture. The problem may then be treated as a classification concern with 3 convolution layers and 2 completely interconnected layers with a final output layer. Estimating the exact age by regression is a challenging process. Age prediction systems have been growing rapidly in recent days thanks to its important modules and use for many computer vision applications, such as interaction between human and computer, safety systems and visual monitoring. The value of an age prediction is shown by several examples. For example, there is an age to get alcohol, drive vehicles, travel alone outside the country, smoke cigarettes, etc. The problem is, however, that human capacities are poor and unreliable in age prediction. So it would be necessary to reject underage individuals with computer vision systems. Hotels, airports, busses, casinos, government buildings, universities, hospitals, movie theatres, etc. are currently using automated age and gender prediction systems for improving protection and mitigating possible threats or poverty.Age prediction methods are also used in healthcare systems, knowledge recovery, academic studies, and Electronic Customer Relationship Management (ECRM) applications, which distributes customers to a range of aged groups including teenagers, teens, adults and senior citizens. 
Moreover, it may allow businesses to identify products and services according to their age groups that increase income and make more money to collect those customers' daily lives information including behaviors, preferences, practices, 4 priorities etc.For instance, clothing shops that sell men's or women's fashion according to their age groups; restaurants wish to know the most common meals per group of age; many businesses want those audiences to advertise according to their age groups.

\section{LITERATURE SURVEY}

A new architecture for face image classification named unsupervised CNN was introduced by S. U. Rehman et al. [2]. A CNN that handles multitask (i.e. Facial detection and emotional classification) is made by merging $\mathrm{CNN}$ with other modules and algorithms. A hybrid deep CNN and RNN (Recurrent Neural Network) model was introduced by N. Jain et al. [4]. This model aims to improve the overall result of face detection. MI Facial Expression and JAFFE dataset were used to evaluate the model. A convolutional network architecture was proposed by G. Levi et al. [5] that classified the age with small amounts of data. The Audience Benchmark was used to train the model. A system in which a real time automatic facial expression system was designed was proposed by $\mathrm{S}$. Turabzadeh et al. [6]. It was implemented and tested on an embedded device which could be the first step for a specific facial expression recognition chip for a social robot. MATLAB was first used to build and simulate the system and then it was built on an embedded system. The hardship of performing automatic prediction of age, gender and ethnicity on the East Asian Population using a Convolutional Neural Network (CNN) was explored by N. Srinivas et al. [3]. A fine-grained ethnicity has predictions based on a refined categorization of the human population (Chinese, Japanese, Korean, etc.). Previous results suggest that the most critical job is to predict the fine-grained ethnicity of a person, followed by age and lastly gender. An automated recognition system for age, gender and emotion was presented by A. Dehghan et al. [7] that was trained using deep neural network. At the ImageNet LSVRC-2010 contest, A. Krizhevskyetal. [8] presented a paper which suggested segregation of 1.2 million images into 1000 different categories with the help of a deep Convolutional neural network. The results which were obtained suggested that supervised learning can deliver exceptional accuracies. Some datasets have annotations on the face images which are not considered to be of any use for face recognition. Some papers have also used RNN but it is not applicable for our project as the RNN takes text or speech as an input whereas we required an image to be as the input. Hence, CNN is chosen over RNN for the sake of our project. Some papers also suggest the use of unsupervised CNN, but, for this project supervised learning is more appropriate. The UTKFace dataset is used as dataset for the project.

\section{SOFTWARE REQUIREMENTS AND TECHNOLOGIES}

To predict the age, we are going to use a convolutional neural Architecture of the network (CNN). This CNN uses 3 layers of convolution and 2 layers with one final output layer.

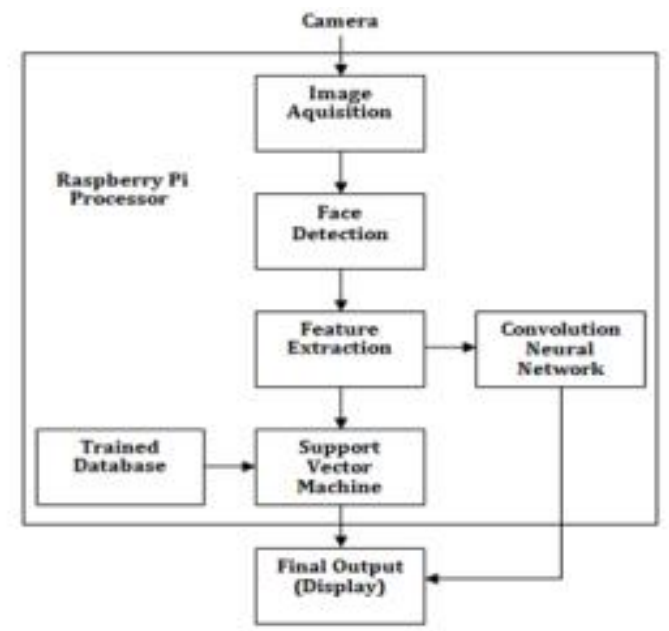

This problem can be interpreted rather than regression as a classification problem. It is a difficult job to estimate the exact age by means of regression. Simply by looking at the face even people can not predict age. In an age range, like 20-30 or 30-40, we will therefore seek to predict the age. It is hard to predict how a person's age depends on many factors from a single image.

\section{ANALYSIS}

Age detection is the process of automatically discerning the age of a person solely from a photo of their face. Typically, you'll see age detection implemented as a two-stage process:

1. Stage \#1:Detect faces in the input image/video stream

2. Stage \#2:Extract the face Region of Interest (ROI), and apply the age detector algorithm to predict the age of the person

For Stage \#1, any face detector capable of producing bounding boxes for faces in an image can be used

Once your face detector has produced the bounding box coordinates of the face in the image/video stream, you can move on to Stage \#2 - identifying the age of the person.

\section{MODULES}

- NUMPY: Numpy is the most basic yet a powerful package for mathematical and scientific computing and data manipulation in python. It is an open source library available in python.

- PANDAS: Pandas library is used for data manipulation and analysis. It supports reading and writing excel spreadsheets, CVS's and whole lot of manipulation.

- CV2: OpenCV is a high performance library for digital image processing and computer vision, which is free and open source.

- MATPLOTLIB: Matplotlib is a plotting library for the python programming language and its numerical mathematics extensions in numpy.

- OS: The OS module in python provides a way of using operating system dependent functionality.

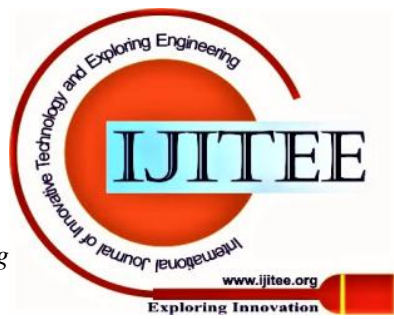


- PIL: PIL library is used for image manipulation in python. Using this library we are opening an image from dataset and resizing it.

- SCIPY : SciPy library contains different modules for optimization, linear algebra, integration and statistics.

- X KERAS : Keras is an open-source high-level neural network API, written in python. It allows easy and fast prototyping

- TENSORFLOW : Tensorflow is a free and opensource software library for dataflow and differentiable programming across a range of tasks. It is used in neural networks.

\section{TECHNOLOGIES}

- Image Processing: Image processing is a method to perform some operations on an image, in order to get an enhanced image or to extract some useful information from it. It is a type of signal processing in which input is an image and output may be image or characteristics/features associated with that image. Nowadays, image processing is among rapidly growing technologies. It forms core research area within engineering and computer science disciplines too.

Image processing basically includes the following three steps :

Importing the image via image acquisition tools;

Analysing and manipulating the image;

Output in which result can be altered image or report that is based on image analysis.

There are two types of methods used for image processing namely, analogue and digital image processing. Analogue image processing can be used for the hard copies like printouts and photographs. Image analysts use various fundamentals of interpretation while using these visual techniques. Digital image processing techniques help in manipulation of the digital images by using computers. The three general phases that all types of data have to undergo while using digital technique are pre-processing, enhancement, and display, information extraction.

- Computer Vision : Computer vision is a field of artificial intelligence that trains computers to interpret and understand the visual world. Using digital images from cameras and videos and deep learning models, machines can accurately identify and classify objects and then react to what they "see."

\section{SOFTWARE DESIGN}

\section{- UML DIAGRAMS}

A UML diagram is a diagram with the purpose of visually representing a system along with its main actors, roles, actions, artifacts or classes, in order to better understand, alter, maintain, or document information about the system.

\section{- CLASS DIAGRAM}

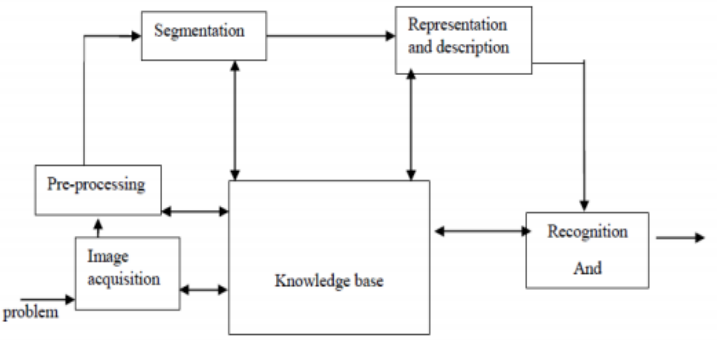

Fig. 1: Class Diagram

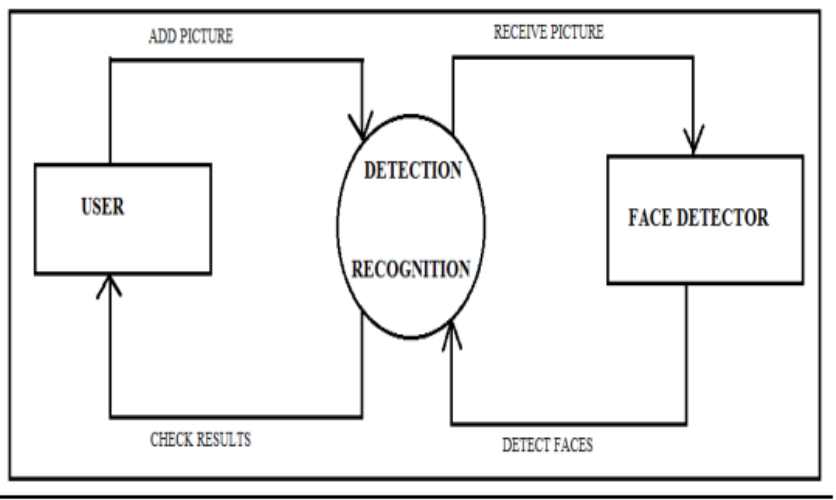

Fig. 2: Use Case Diagram
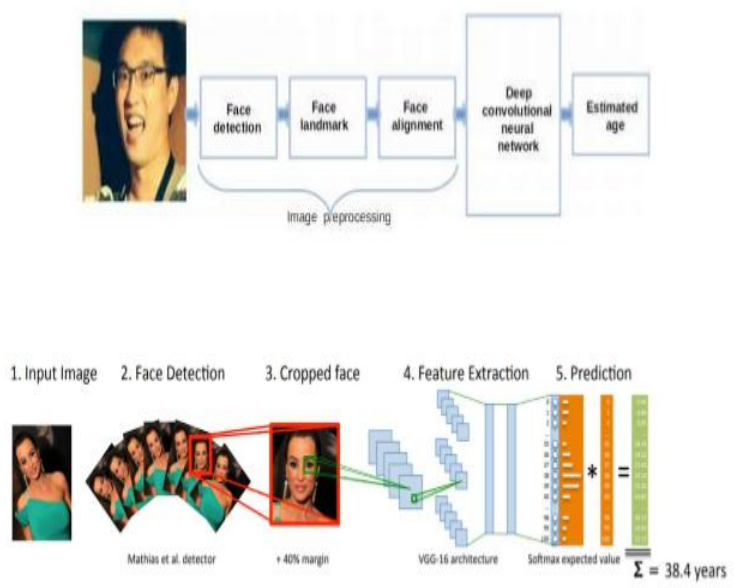

Fig. 3. Data Flow Diagram 
User Module:

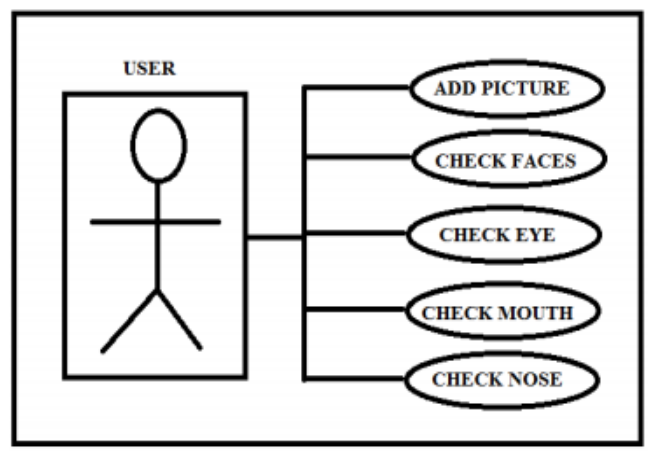

Software Module:

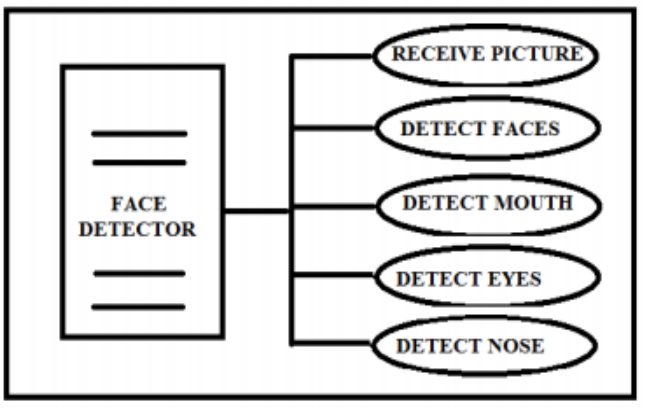

Fig. 4. Design

The ideal approach is Age Prediction, since we expect an actual number as the output. However, it is difficult to estimate age with precise regression. And people can not determine age on the basis of a person's views precisely. Nonetheless, in their twenties or thirties we have an idea. It is therefore prudent to regard this topic as a classifying topic in which we seek to determine the age group in which the individual is present. For instance, in the 0-2 range is a single grade, 4-6 is a different grade etc. This must be borne in mind that estimating an age with a single image is not easy to solve as it depends on various variables, and in different parts of the world, the people of the same age will look very different.

In addition, before making predictions, we evaluated the use of face alignment and found that the predictions had improved for some cases but that they were worse for some people. When you mainly deal with non-frontal ears, it may be a smart idea to use alignment.

\section{IMPLEMENTATION AND RESULT ANALYSIS}

$\begin{array}{cc}\text { HARDWARE REQUIREMENTS } \\ \text { - System } & : \text { Intel i5 } 2.1 \mathrm{GHZ} \\ \text { - Memory } & : \text { : } 4 / 8 \mathrm{~GB} . \\ \text { - Hard Disk } & : \text { : } 1 \mathrm{~TB}\end{array}$

\section{SOFTWARE REQUIREMENTS}

- Operating System : Windows 7/8 and above

- Domain

- Scripts

- Tool Machine Learning Python Anaconda Navigator, Jupiter

Notebook IDE

- Libraries : Numpy, pandas, math, cv2, matplotlib, seaborn, os, Image, scipy, sklearn, keras and tensorflow

\section{DESCRIPTION OF LIBRARIES USED}

NUMPY:NumPy is a library for the Python programming language, adding support for large, multidimensional arrays and matrices, along with a large collection of high-level mathematical functions to operate on these arrays.

PANDAS:Pandas is a software library written for the Python programming language for data manipulation and analysis. In particular, it offers data structures and operations for manipulating numerical tables and time series.

MATPLOT LIBRARIES : Matplotlib is an amazing visualization library in Python for 2D plots of arrays. Matplotlib is a multi-platform data visualization library built on NumPy arrays and designed to work with the broader SciPy stack.

KERAS : Keras is an open-source neural-network library written in Python. It is capable of running on top of TensorFlow, R, Theano, or PlaidML. Designed to enable fast experimentation with deep neural networks, it focuses on being user-friendly, modular, and extensible.

TENSORFLOW : TensorFlow is a free and open-source software library for dataflow and differentiable programming across a range of tasks. It is a symbolic math library, and is also used for machine learning applications such as neural networks.

\section{TOOLS USED}

Anaconda Navigator : Anaconda is a free and open-source distribution of the Python and $\mathrm{R}$ programming languages for scientific computing (data science, machine learning applications, large-scale data processing, predictive analytics, etc.), management and deployment.

Jupyter Notebook IDE: Jupyter Notebook is an opensourced web-based application which allows you to create and share documents containing live code, equations, visualisations, and narrative text. The IDE also includes data cleaning and transformation, numerical simulation, statistical modelling, data visualisation, and many others.

Steps to follow:

1. Face detection with Haar cascade

2. Age Recognition with CNN

\section{Face detection with Haar cascades:}

This is a part most of us at least have heard of. OpenCV/JavaCV provide direct methods to import Haarcascades and use them to detect face

\section{Age Recognition with $\mathrm{CNN}$}

CNN algorithm is used for age recognition. The CNN's output layer (probability layer) in this $\mathrm{CNN}$ consists of 5 values for 5 age classes ("1-14", "14-25”, “25-40”, “40$60 "$, “60-"). 


\section{Procedure:}

- First we are changing the current directory to the path where our image dataset is been stored by using os.chdir( ) and then we get the list of all the files and directories in the specified path using os.listdir( ).

- Shuffle( ) is used to randomize all the image files.

- Using split( ) we get the ids of image files and then store it in age variable.

- A list named classes is created to store ages less than 14 as 0 , between 14 and 25 as 1,between 25 and 40 as 2, between 40 and 60 as 3 and above 60 as 4 .

- Using misc.imread( ) and cv2.resize( ), we read an image from each file as an array and resize its dimensions to $32 \times 32$ and then store it in a list names X_data.

- Using squeeze( ), we remove single-dimensional entries from shape of array and store in the variable $\mathrm{X}$.

- Next we normalize the data by converting the datatype of variable $\mathrm{X}$ to float 32 and dividing it by 255.

- $\quad$ Slice the list classes up to 10 items and convert class vector(integers) to binary class matrix using to_categorical( ) before training our model.

- The total length of dataset is 23708 , out of which 15008 is used for training, 1700 for testing and 7000 for validating the data.

- $\quad$ There are two ways to build keras models: sequential and functional. In our project we are using sequential by which we create a model layer-by-layer.

- When we use Conv2D as the first layer we must define input shape. This layer creates a convolution kernel that is convolved with the layer input to produce a tensor of outputs.

- MaxPooling2D layer is used for spatial data.

- Dropout layer is used to prevent a model from overfitting.

- Dense layer is a linear operation in which every input is connected to every output by a w eight.

- We summarize the model by using model.summary( ). Total params: 534,885, trainable params: 534,885 and Non-trainable params: 0

- Next we compile and fit the model.

- We then evaluate the model on test set to get the accuracy up to 0.6170588 .

- Now the model is trained and its ready to predict the age of any random image from dataset.

- We plot a random sample of 10 test images, their predicted labels and ground truth.

- We get the output by displaying each image along with its title.

In this project, Keras is used to work on Tensorflow. Keras is an open-source neural network library. It is userfriendly and provides several features such as activation functions, layers, optimizers, etc. and it supports CNN . By using the appropriate class in Keras, deep learning models can be created on iOS and Android through the JVM (Java Virtual Machine). Keras enables the model to perform random transformations and normalization operations batches of image data by working on different attributes such as height shift, width shift, rotation range, rescale, range of shear, range of zoom, horizontal rip and fill mode. Using these attributes the system can automatically rotate, translate, rescale, and zoom into or out of images, as well as apply shearing transformations, rip images horizontally, fill in newly created pixels, etc. For the purpose of image classification, ConvNet is used.

Training Dataset: The training dataset is used as a set of examples used for training the model, i.e. to fit the different parameters.

Validation Dataset: A validation dataset is used to fit the hyper-parameters of the classifier. A validation dataset is necessary because it helps in the reduction of overfitting. The validation dataset is independent of the trainingdataset.

Test Dataset: The test dataset is used to test the performance of the classifier or model and to check the performance of characteristics such as accuracy, loss, sensitivity, etc. It is independent of the training and validation dataset.

\section{MODEL FOR TRAINING THE DATA SET}

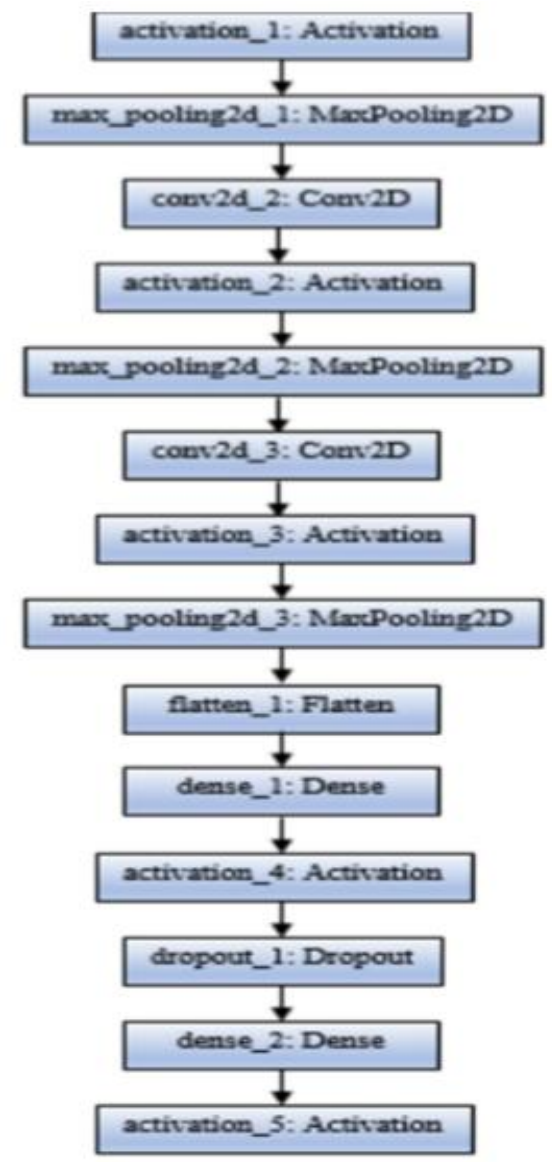




\section{Age Prediction using Image Dataset using Machine Learning}

\section{SYSTEM TESTING}

System testingis a level of testing that validates the complete and fully integrated software product. The purpose of a system test is to evaluate the end-to-end system specifications. Usually, the software is only one element of a larger computer-based system. Ultimately, the software is interfaced with other software/hardware systems. System Testing is actually a series of different tests whose sole purpose is to exercise the full computer-based system.

- The total length of dataset is 23708 , out of which 15008 is used for training,1700 for testing and 7000 for validating the data.

- Variations in shape: One image had a shape $(66,46)$ whereas other had $(102,87)$.

- Multiple viewpoints: We have faces with whichever view possible.

- The system is tested based on all the possible angles of images and problems which may occur.

The proposed system works quite well with all the testing conditions mentioned below with pretty well accuracy.

\section{TEST CASE 1: side view}

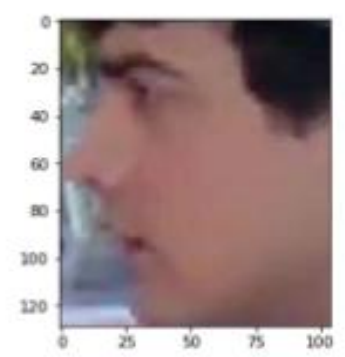

\section{TEST CASE 2: front view}

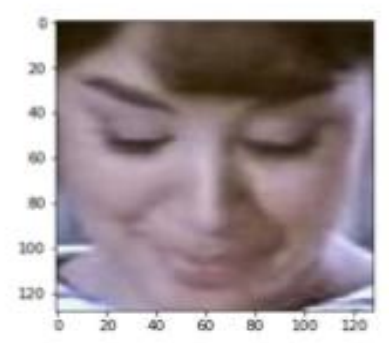

TEST CASE 3: Quality of images

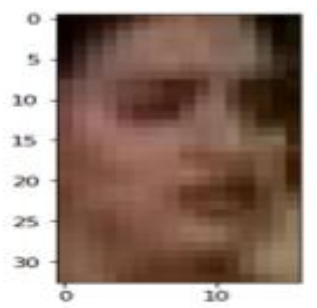

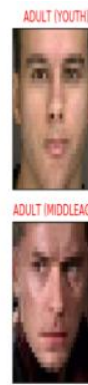
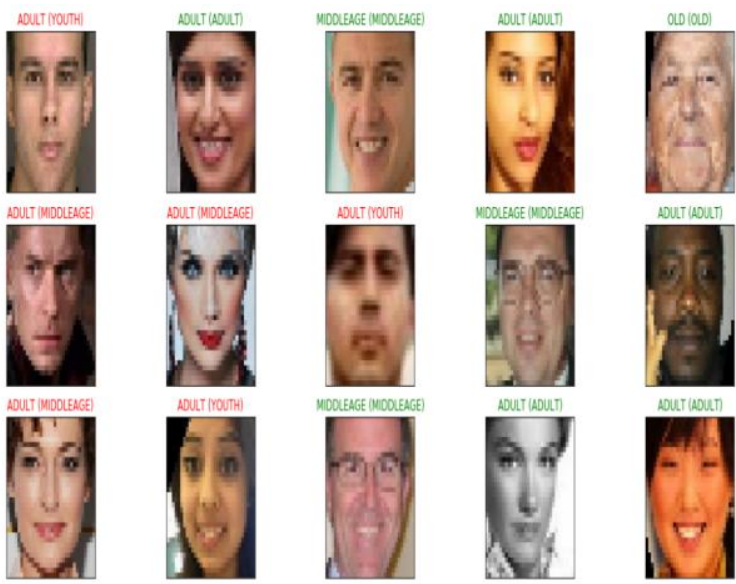

\section{CONCLUSION AND FUTURE WORK}

The model proposed was developed very carefully and error-free while being efficient. During this research, we proposed a model to estimate people's age by feeding the CNN image dataset, a deep learning algorithm and trained in broad database face-recognition. In all, we think that the accuracy of the model is decent and better than many already existing model, but can be further improved by using more data, data increase and better network architecture. The project model also predicts the age of the image provided with little slip and angle issue.The completely automated face recognition program was not sufficiently reliable to achieve high accuracy of recognition. It was mainly due to the fact that even a slight invariance to the size, rotation or shift errors of the segmented facial image did not occur in the face recognizing subsystem.

This project allows us to obtain useful knowledge about a variety of topics such as deep learning, the use of different libraries such as Keras, Pil, Seaborn, Tensorflow. The entire model is protected and this project has also enabled us to understand the stages of a project's creation and the working together. We have also learned how to test various project features. This project has given us great pleasure in creating a concept that can be used for good purposes and health in real life. In our project, there is ample scope for further development. For addition, a variety of features such as gender and age can be applied to this program. Outside of classification for age prediction, a regression model may also be used, if enough data is available. Through developing this project further, camera footage for safety purposes can be used for real-time age prediction.Nevertheless, if a more processing such as an eye detection technology has been applied to further normalize the segmented facial image, the output will expand to levels comparable to the manual facial detection and recognition system. This is one of the system needs identified in this section. Good techniques such as iris or retina recognition and facial recognition are used for user access and user authentication applications using the thermal range as this requires very high precision. The automatic real time system will be perfect for crowd control application.

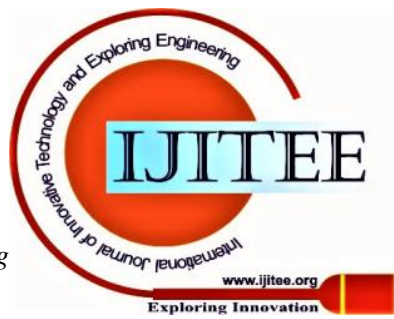


Invariant face detection and recognition systems.In order to be used in simple surveillance applications, such as ATM user security, the fully automated face detection and recognition System (with an eyeshoot detection system) could be applied, whereas manual face detection and an automated recognition system is ideal for the mug shot matching. Implementation of a technique for eye detection would be a small extension to the system implemented and require little additional investigation. All other methods have shown good results and rely on the deformable prototype and main component analysis strategies.

\section{REFERENCES}

1. Dataset downloaded from Kaggle website: https://www.kaggle.com/age-groupclassification- with-cnn

2. S. U. Rehman, S. Tu, Y. Huang, and Z. Yang, Face recognition: A Novel Un-supervised Convolutional Neural Network Method, IEEE International Conference of Online Analysis and Computing Science (ICOACS), 2016

3. N. Srinivas, H. Atwal, D. C. Rose, G. Mahalingam, K. Ricanek, and D. S. Bolme, Age, Gender, and Fine-Grained Ethnicity Prediction Using Convolutional Neural Networks for the East Asian Face Dataset, 12th IEEE International Conference on Automatic Face and Gesture Recognition (FG 2017), 2017.

4. N. Jain, S. Kumar, A. Kumar, P. Shamsolmoali, and M. Zareapoor, Hybrid Deep Neural Networks for Face Emotion recognition, Pattern Recognition Letters, 2018.

5. G. Levi, and T. Hassner," Age and Gender Classification Using Convolutional Neural Networks," IEEE Workshop on Analysis and Modeling of Faces and Gestures (AMFG), IEEE Conf. on Computer Vision and Pattern Recognition (CVPR), Boston, 2015.

6. S. Turabzadeh, H. Meng, R. M. Swash, M. Pleva, and J. Juhar, Realtime Emotional State Detection From Facial Expression On Embedded Devices, Seventh International Conference on Innovative Computing Technology (INTECH), 2017.

7. A. Dehghan, E. G. Ortiz, G. Shu, and S. Z. Masood, Dager: Deep Age, Gender and Emotion Recognition Using Convolutional Neural Network, arXiv preprint arXiv: 1702.04280, 2017.

8. A. Krizhevsky, I. Sutskever, and G. E. Hinton, ImageNet classification with deep convolutional neural networks, Communications of the ACM, vol. 60, no. 6, pp. 8490, 2017 